\title{
ESTIMATION OF DIRECTION-OF-ARRIVAL AND POLARIZATION WITH DIVERSELY POLARIZED ANTENNAS IN A CIRCULAR SYMMETRY INCORPORATING MUTUAL COUPLING EFFECTS.
}

\author{
Roald Goossens and Hendrik Rogier \\ INTEC, Ghent University St-Pietersnieuwstraat 41, 9000 Gent Belgium,Email: Roald.Goossens@intec.UGent.be and \\ Hendrik.Rogier@intec.UGent.be
}

\begin{abstract}
A modified Root-MUSIC algorithm is proposed to estimate the directions-of-arrival and the polarization parameters of plane waves using a diversely polarized uniform circular array. Special attention is devoted to the presence of the mutual coupling effects. A well chosen phase-mode expansion for the induced voltages over the antenna elements in combination with the symmetry of the array enables us to develop a computationally efficient estimation algorithm which incorporates all electromagnetic effects. Finally, the efficiency of the new algorithm is verified based on synthetic antenna data.
\end{abstract}

Key words: polarization, DOA estimation, mutual coupling.

\section{INTRODUCTION}

The system capacity of wireless systems can be significantly increased by deploying smart antenna configurations. Applications like beamforming and direction-ofarrival estimation make it possible to mitigate strong interferences and thus improve the performance of the mobile communication system.

Techniques for estimating the directions-of-arrival (DOAs) of signals impinging on an antenna array have been the subject of intensive study. In the majority of cases uniformly polarized arrays are employed. The present paper proposes a modified Root-MUSIC algorithm which estimates the DOAs and polarization of the impinging plane waves using a uniform circular array (UCA) with diversely polarized antennas. Although the use of diversely polarized antennas is rarely studied $[1,2,3]$ to estimate DOAs, using these types of antennas in an array configuration has some advantages over uniformly polarized arrays, since they are capable of discriminating signals based on their polarization characteristics. In previous papers algorithms were developed for DOA estimation, with an ULA (uniform linear array) [2] or an L-shaped array [3], based on the
Multiple Signal Classification (MUSIC) algorithm [4]. In the presence of mutual coupling effects, e.g. introduced by nearby scatterers or nearby antenna elements, the traditional algorithms fail to deliver accurate DOA estimates. Uniform circular arrays enable us to incorporate mutual coupling into DOA estimation algorithms due to their circular symmetry. In [5] it is proven that by the expansion of the open-circuit voltages into a limited number of phase modes the MUSIC algorithm can be adapted to incorporate mutual coupling effects. In [6] this expansion is extended to estimate the azimuth angle as well as the elevation angle with UCAs consisting of uniformly polarized elements. In this paper the expansion of the induced voltages into phase modes is applied for UCAs consisting of diversely polarized antennas. Because of this, a time-efficient algorithm is obtained by first estimating the azimuth angle of arrival by means of polynomial rooting and then determining the polarization for each azimuth angle estimate. By means of the Cramer Rao Bound (CRB) we prove that the proposed Root-MUSIC algorithm is an efficient DOA estimation algorithm and that UCAs consisting of diversely polarized antennas are capable to more accurately estimating the elevation angle than UCAs consisting of uniformly polarized elements.

The structure of the paper is as follows. In Section 2 the electromagnetic characteristics of the array with diversely polarized antennas are described when an arbitrary elliptical polarized plane wave impinges on the array. This rigorous electromagnetic description of the UCA enables us to adapt the Root-MUSIC algorithm to our purposes in section 3 and to estimate the DOAs as well as the orientation and the shape of the polarization ellipse. In section 4 the proposed algorithm is tested with some illustrative examples based on synthetic antenna data generated by means of the well-established NEC2-code.

\section{RECEIVING CHARACTERISTICS OF UCA}

We consider an array with $P$ identical antenna elements as shown in Fig. 1. These elements are uniformly distributed over a circle with radius $R$. The phase center of each 
antenna element is located in the $x y$-plane, at azimuth angles $\varphi_{t}=(t-1) \frac{2 \pi}{P}$ and the antennas are oriented in such a way that circular symmetry is obtained. When $K$ plane

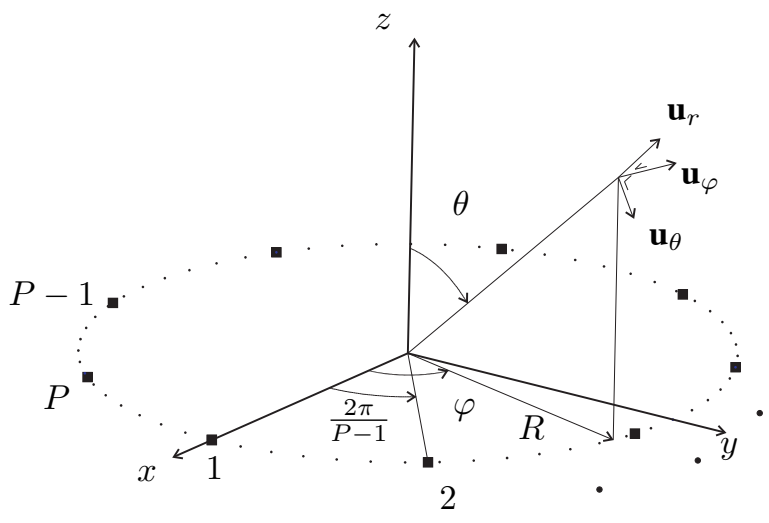

Figure 1. uniform circular array composed of $P$ antenna elements.

waves impinge on the UCA, the signal model of this array is given by

$$
\mathbf{x}(t)=\mathbf{A}(\theta, \varphi, \gamma, \eta) \mathbf{s}(t)+\mathbf{n}(t),
$$

where $\mathbf{A}(\theta, \varphi, \gamma, \eta)$ is formed by the set of the $K$ element space manifold vectors $\mathbf{a}\left(\theta_{k}, \varphi_{k}, \gamma_{k}, \eta_{k}\right)(k=1 \ldots K)$. The element space manifold vector $\mathbf{a}(\theta, \varphi, \gamma, \eta)$ contains the voltages induced over all antennas when a plane wave $\mathbf{E}=E \sin \gamma e^{j \eta} \mathbf{u}_{\theta}+E \cos \gamma \mathbf{u}_{\varphi}$ impinges on the array from $\operatorname{DOA}(\theta, \varphi)$,

$$
\begin{array}{r}
\mathbf{a}(\theta, \varphi, \gamma, \eta)=\left[a_{1}(\theta, \varphi, \gamma, \eta), a_{2}(\theta, \varphi, \gamma, \eta), \ldots,\right. \\
\left.a_{P-1}(\theta, \varphi, \gamma, \eta), a_{P}(\theta, \varphi, \gamma, \eta)\right]^{T} .
\end{array}
$$

Because the antenna elements of the UCA are diversily polarized, this vector does not only depend on the DOA $(\theta, \varphi)$, but also depends on the polarization parameters of the plane wave $(\gamma, \eta)$, the auxiliary polarization angle and the polarization phase difference, respectively. Hence, the element space manifold vector can be divided into a vertical and horizontal contribution:

$$
\mathbf{a}(\theta, \varphi, \gamma, \eta)=\sin \gamma e^{j \eta} \mathbf{a}_{\theta}(\theta, \varphi)+\cos \gamma \mathbf{a}_{\varphi}(\theta, \varphi) .
$$

where $\mathbf{a}_{\theta}(\theta, \varphi)$ and $\mathbf{a}_{\varphi}(\theta, \varphi)$ are the collection of the voltages which are induced when respectively a vertical and a horizontal polarized plane wave with a unit amplitude impinges on the array at DOA $(\theta, \varphi)$. Thanks to the circular symmetry in the UCA the voltages induced over the different antenna elements are related to each other:

$$
a_{t}(\theta, \varphi, \gamma, \eta)=a_{1}\left(\theta, \varphi-\varphi_{t}, \gamma, \eta\right) .
$$

It is clear that as a result of mutual coupling the receiving properties of the antenna elements are different in relation to the stand-alone characteristics. On the one hand terminal currents induced in the loads of neighboring elements introduce an extra voltage contribution in the antenna element under consideration. On the other hand the radiation pattern of the stand-alone antenna element is deformed due the detailed current distributions induced in the opencircuited neighboring antenna elements (shadowing effects) or neighboring scatterers (platform effects). Although these mutual coupling effects complicate the receiving properties of the UCA, it is proven [5] that, even in the presence of other antenna elements, the induced voltage over an antenna element can be expanded into a limited number of phase modes in the azimuth angle $\varphi$ :

$$
a_{1}(\theta, \varphi, \gamma, \eta)=\sum_{m=-M}^{+M} a_{m}(\theta) e^{j m \varphi}
$$

The expansion is only limited by the dimensions of the UCA, $M>\frac{2 \pi d}{\lambda}$, with $d$ the largest dimension of the array and $\lambda$ the wavelength. Based on the theory outlined in [8], following rule of thumb can be derived in order to obtain an accurate description of all mutual coupling effects better than $0.1 \%$ :

$$
M=\left\lceil 4 \frac{d}{\lambda}+4\right\rceil
$$

with $\lceil\ldots\rceil$ the ceiling operation. In the case of an UCA, consisting of diversely polarized antennas, Eq. 5 becomes

$$
\begin{array}{r}
a_{1}(\theta, \varphi, \gamma, \eta)=\sin \gamma e^{j \eta} \sum_{m=-M}^{+M} a_{m}^{\theta}(\theta) e^{j m \varphi}+ \\
\cos \gamma \sum_{m=-M}^{+M} a_{m}^{\varphi}(\theta) e^{j m \varphi},
\end{array}
$$

where the vertical component as well as the horizontal component of the induced voltage is expanded into a limited number of phase modes. Together with Eq. 4 the receiving properties, including mutual coupling, are determined by only $2 M+1$ phase modes $\left\{a_{m}^{\theta}(\theta)\right\}$ and $\left\{a_{m}^{\varphi}(\theta)\right\}$.

\section{DOA AND POLARIZATION ESTIMATION}

In section 2 a compact description of the receiving characteristics is given which incorporates all mutual coupling effects. This compact description enables a fast and efficient estimation of the DOAs and polarization parameters. Consider $K$ plane waves which impinge at a fixed elevation angle $\theta_{0}$. The signal model of the array is given by Eq. 1 where

$$
\begin{aligned}
& \mathbf{A}=\left[\mathbf{a}\left(\theta_{0}, \varphi_{1}, \gamma_{1}, \eta_{1}\right), \mathbf{a}\left(\theta_{0}, \varphi_{2}, \gamma_{2}, \eta_{2}\right), \ldots,\right. \\
& \left.\mathbf{a}\left(\theta_{0}, \varphi_{K-1}, \gamma_{K-1}, \eta_{K-1}\right), \mathbf{a}\left(\theta_{0}, \varphi_{K}, \gamma_{K}, \eta_{K}\right)\right] .
\end{aligned}
$$

In a first step the measured data is subjected to a beamspace transformation [7]:

$$
\begin{gathered}
\mathbf{x}_{\text {beam }}(t)=\mathbf{W} \mathbf{x}(t), \\
{[\mathbf{W}]_{s, t}=\frac{1}{\sqrt{K}} e^{j \frac{2 \pi}{P}(s-M-1)(t-1)}}
\end{gathered}
$$


with $t=1 \ldots P$ and $s=1 \ldots 2 M+1$. For DOA estimation we calculate the beamspace data covariance matrix and the eigendecomposition is

$$
\mathbf{R}=E\left\{\mathbf{x}_{\text {beam }}(t) \mathbf{x}_{\text {beam }}^{H}(t)\right\}=E_{S} \Lambda_{S} E_{S}^{H}+E_{N} \Lambda_{N} E_{N}^{H} .
$$

The diagonal matrices $\Lambda_{N} \in \mathbb{R}^{2 M+1-K \times 2 M+1-K}$ and $\Lambda_{S} \in \mathbb{R}^{K \times K}$ contain the noise-subspace and signalsubspace eigenvalues of $\mathbf{R}$, respectively. The columns of the matrices $E_{N} \in \mathbb{C}^{2 M+1 \times 2 M+1-K}$ and $E_{S} \in$ $\mathbb{C}^{2 M+1 \times K}$ denote the corresponding noise-subspace and signal subspace eigenvectors. The beamspace MUSIC algorithm estimates the signal DOAs and polarization from the $K$ deepest nulls of the MUSIC function

$$
\begin{aligned}
& f_{\text {music }}\left(\theta_{0}, \varphi, \gamma, \eta\right)= \\
& \quad \mathbf{a}_{\text {beam }}^{H}\left(\theta_{0}, \varphi, \gamma, \eta\right) E_{N} E_{N}^{H} \mathbf{a}_{\text {beam }}\left(\theta_{0}, \varphi, \gamma, \eta\right)
\end{aligned}
$$

with $\mathbf{a}_{\text {beam }}\left(\theta_{0}, \varphi, \gamma, \eta\right)=\mathbf{W a}\left(\theta_{0}, \varphi, \gamma, \eta\right)$. Due to the beamspace transformation the polarization parameters $(\gamma, \eta)$ and the azimuth angle $\varphi$ can be separated from each other. The beamspace array manifold is written by:

$$
\mathbf{a}_{\text {beam }}\left(\theta_{0}, \varphi, \gamma, \eta\right)=\mathbf{M}(z) \mathbf{q}(\gamma, \eta)
$$

with

$$
\mathbf{M}(z)=\left[\begin{array}{lll}
a_{-M}^{\theta}\left(\theta_{0}\right) z^{-M} & \ldots & a_{M}^{\theta}\left(\theta_{0}\right) z^{M} \\
a_{-M}^{\varphi}\left(\theta_{0}\right) z^{-M} & \ldots & a_{M}^{\varphi}\left(\theta_{0}\right) z^{M}
\end{array}\right]^{T},
$$

and

$$
\mathbf{q}(\gamma, \eta)=\left[\begin{array}{c}
\sin \gamma e^{j \eta} \\
\cos \gamma
\end{array}\right] \quad, \quad z=e^{j \varphi} .
$$

The beamspace operation exploits symmetry in the UCA and expands the array manifold into separate phase modes. The expansion Eq. 13 is only legitimate provided

$$
P>2 M+1,
$$

otherwise the phase modes mix up. Substituting Eq. 13 in Eq. 12 yields

$$
\begin{aligned}
& f_{\text {music }}\left(\theta_{0}, \varphi, \gamma, \eta\right)= \\
& \mathbf{q}^{H}(\gamma, \eta) \mathbf{M}^{H}(z) \mathbf{E}_{N} \mathbf{E}_{N}^{H} \mathbf{M}(z) \mathbf{q}(\gamma, \eta) .
\end{aligned}
$$

Our aim is to first estimate the azimuth angles $\varphi$ independent from the polarization of the incoming waves. Therefore, we note that, for every value of $\varphi$, the function Eq. 17 is minimized when $\mathbf{q}(\gamma, \eta)$ is equal to the eigenvector corresponding to the smallest eigenvalue of the $2 \times 2$ matrix

$$
\mathbf{C}(z)=\mathbf{M}^{H}(z) \mathbf{E}_{N} \mathbf{E}_{N}^{H} \mathbf{M}(z) .
$$

The smallest eigenvalue in Eq. 18 is always nonnegative, and equal to zero (or close to zero in the presence of noise) when $\varphi$ equals one of the observed DOA's. Hence, the aim is to find values of $z$ for which

$$
\operatorname{det}\{\mathbf{C}(z)\}=0 \text {. }
$$

The condition Eq. 19 is a polynomial in $z$, so estimating the DOAs is transformed into polynomial rooting (RootMUSIC). The roots which are located close to the unit circle deliver estimates for the azimuth angles, i.e. $\widehat{\varphi}=$ $\angle \frac{z}{|z|}$. To estimate the polarization parameters, we again consider the MUSIC function evaluated in the estimated azimuth angles:

$$
\begin{aligned}
f_{\text {music }}\left(\theta_{0}, \widehat{\varphi}, \gamma, \eta\right) & =\left|\mathbf{E}_{N}^{H} \mathbf{M}\left(e^{j \varphi}\right) \mathbf{q}(\gamma, \eta)\right|^{2} \\
& =\mathbf{q}^{H}(\gamma, \eta) \mathbf{C}\left(e^{j \varphi}\right) \mathbf{q}(\gamma, \eta) .
\end{aligned}
$$

This function has a zero value for the following values of the polarization parameters:

$$
\begin{gathered}
\widehat{\gamma}=\arctan \frac{\left|C_{12}\right|}{\left|C_{11}\right|}, \\
\widehat{\eta}=\pi-j \ln \left(\frac{C_{12}}{\left|C_{12}\right|} \cdot \frac{\left|C_{11}\right|}{C_{11}}\right) .
\end{gathered}
$$

Additional or spurious solutions can be created by Eq. 19 . By calculating the MUSIC function for every estimate $(\widehat{\varphi}, \widehat{\gamma}, \widehat{\eta})$ it is possible to reject these spurious solutions because only the $K$ estimates that result in values of the MUSIC function closest to zero are accepted.

\section{EXAMPLES}

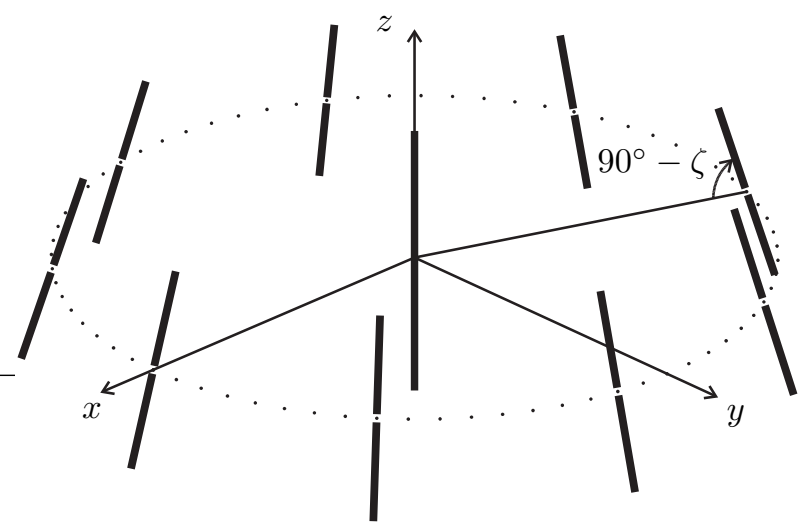

Figure 2. UCA consisting of nine dipole elements tilted over $\zeta$ with a short-circuited dipole in the center of the $U C A$.

In order to validate our new approach, we consider a uniform circular array of nine dipole antenna elements tuned to $900 \mathrm{MHz}$. All results are obtained based on synthetic data generated by means of the well-established NEC-2 code. The array elements are distributed uniformly on a circle with diameter $d=\frac{\lambda}{2}(=16.12 \mathrm{~cm})$. In the center of the circle there is a short-circuited dipole with length $d=16.12 \mathrm{~cm}$. The short-circuited dipole acts as a platform effect and introduces severe mutual coupling. Traditional DOA estimation algorithms, such as UCA-RBMUSIC [7], fail to deliver accurate estimates in the presence of this severe mutual coupling. The antenna elements of the UCA are now tilted over an angle $\zeta$ (see 
Fig. 2). Due to this tilt the dipoles become diversely polarized and the UCA is capable of discriminating signals based on their polarization characteristics. A disadvantage of a UCA, built by vertically oriented dipole elements, is that the estimation of the elevation angle is quite inaccurate for plane waves which impinge from elevation angles near $90^{\circ}$, the region of interest in most applications. Tilting the antennas overcomes this problem. In Fig. 3 the Cramer Rao bound (CRB) for the elevation angle $\left(\mathrm{CRB}_{\theta}\right)$ and azimuth angle $\left(\mathrm{CRB}_{\varphi}\right)$ is plotted for several values of $\zeta$. The CRB defines the ultimate accuracy of any estimation procedure in the presence of noise. For $\zeta=0^{\circ}$ we see that $\mathrm{CRB}_{\theta}$ becomes very large when a signal arrives from elevation angles near $\theta=90^{\circ}$. Notice that the CRBs are independent from the azimuth angle due to the circular symmetry of the UCA. By tilting
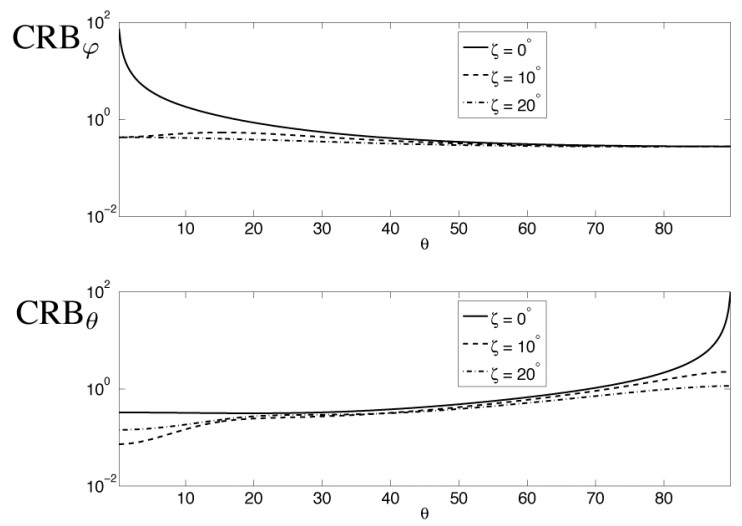

Figure 3. $C R B_{\varphi}$ and $C R B_{\theta}$ for different $\zeta$-values in function of the elevation angle $\theta$.

the antenna elements, $\mathrm{CRB}_{\theta}$ is drastically reduced near $\theta=90^{\circ}$ and so the UCA can more accurately detect signals for elevation angle near $90^{\circ}$ as a result of the diversely polarized antennas. In the case of the azimuth angle, we notice that tilting the antennas does not contribute to more accurate estimates. However, near $\theta=90^{\circ}$ the vertically oriented dipoles deliver already sufficient accurate estimates for the azimuth angle.

Crucial in the proposed algorithm is the beamspace transformation which results in the fact that all phase modes are separated from each other (see Eq. 13) and enables us to estimate the azimuth angle and the polarization parameters in a fast and efficient way. A sufficient number of antenna elements (Eq. 16) are necessary to separate all relevant phase modes. In Fig. 4 the amplitude of the phase modes $a_{m}^{\theta}\left(\theta_{0}\right)$ and $a_{m}^{\varphi}\left(\theta_{0}\right)$ are shown for the considered UCA with a tilted angle $\zeta=20^{\circ}$. We assume that all signals impinge on the array at elevation angle $\theta_{0}=70^{\circ}$. It is clear that nine elements are sufficient to separate all relevant phase modes for $m=-4, \ldots, 4$.

Now consider two uncorrelated signals which impinge at DOAs $\left(\theta_{1}, \varphi_{1}\right)=\left(70^{\circ}, 0^{\circ}\right)$ and $\left(\theta_{2}, \varphi\right)=\left(70^{\circ}, 115^{\circ}\right)$. The first signal is elliptically polarized with polarization parameters $\left(\gamma_{1}, \eta_{1}\right)=\left(70^{\circ}, 50^{\circ}\right)$, while the sec- a)

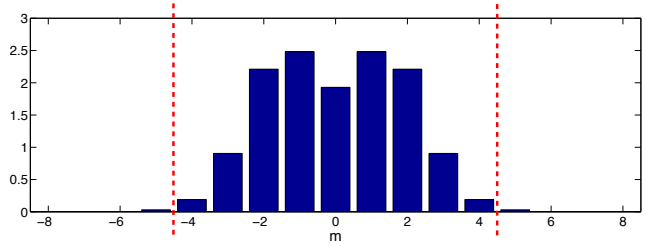

b)

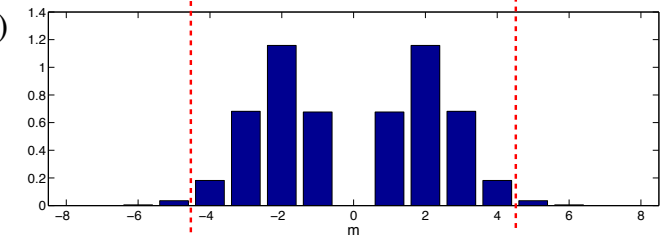

Figure 4. a) phase modes $a_{m}^{\varphi}(\theta)$, b)phase modes $a_{m}^{\theta}(\theta)$ of considered UCA at elevation angle $\theta=70^{\circ}$.

ond signal is linearly polarized with polarization parameters $\left(\gamma_{1}, \eta_{1}\right)=\left(20^{\circ}, 0^{\circ}\right)$. The DOA and polarization of the signals are estimated by means of a 100 bit pseudo-random sequence in the presence of additive white Gaussian noise. In Fig. 5 the root-mean square error (RMSE) of the estimated azimuth angle and of the polarization parameters, of both signals, is plotted for an ensemble consisting of 2000 implementations at different SNR-levels. We assume that both signals have an equal source variance, such that the SNR-level for both signals is equal. We observe that the RMSEs of the different estimates increase when the SNR-level decreases. Besides the RMSEs of the estimates, the corresponding CRBs are also plotted in Fig. 5 and we notice that all RMSEs, except for RMSE of $\eta_{2}$, approach the corresponding CRBs for every value of SNR-level. So we conclude that the algorithm delivers accurate estimates and that it is an efficient algorithm.

a)
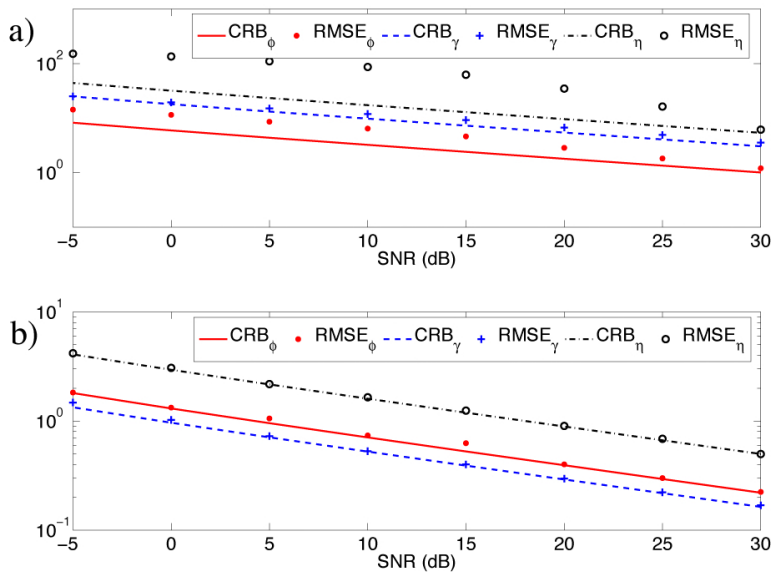

Figure 5. RMSE and CRB for the estimated azimuth angles and polarization parameters of the elliptically polarized signal ( $a$ ) and the linearly polarized signal (b). 


\section{CONCLUSIONS}

In this paper a modified Root-MUSIC algorithm has been developed, which estimates the DOA and the polarization of plane waves. The plane waves impinge on a UCA, which consists of diversely polarized antennas. Special attention is devoted to the presence of mutual coupling which is a major issue in circular arrays. Due to the circular symmetry and the electrical dimensions of the array a limited number of phase modes are sufficient to describe all mutual coupling effects. The expansion of the induced voltages into phase modes enables us to implement a modified Root-MUSIC algorithm. By means of some relevant examples, based on synthetic antenna data, it is proved that this modified Root-MUSIC algorithm yields good estimates for the DOA and polarization.

\section{ACKNOWLEDGMENTS}

H. Rogier is a Postdoctoral Research Fellow of the FWO-V. His research was supported by a grant of the DWTC/SSTC, MOTION project and by a travel grant of the FWO-V.

\section{REFERENCES}

[1] Ferrara ER, Parks TM. Direction finding with an array of antennas having diverse polarizations. IEEE Trans. on Antennas Propag. March 1983; 31-2:231236.

[2] Weiss AJ, Friedlander B. Direction finding for diversely polarized signals using polynomial rooting. IEEE Trans. on Signal Processing May 1993; 412:1893-1905.

[3] Wong KT, Zoltowksi MD. Diversely polarized RootMUSIC for azimuth-elevation angle-of-arrival estimation. Antennas and Propagation Society International Symposium, July 1996; vol. 2, p. 1352-1355.

[4] Schmidt RO. Multiple emitter location and signal parameter estimation. IEEE Trans. Antenn. Propagat. March 1986; 34: 276-280.

[5] Rogier H, Bonek E. Analytical spherical-mode based compensation of mutual coupling in uniform circular arrays for direction-of-arrival estimation. Int. Journal of Electr. and Comm. March 2006; 60-3:179-189.

[6] Goossens R, Rogier H. 2-D direction of arrival estimation combining UCA-RARE and MUSIC for uniform circular arrays subject to mutual coupling. Proc. of International Conference on electromagnetics in advanced applications, Torino, 2005. p.791-794.

[8] Rogier H. Spatial Correlation in Uniform Circular Arrays based on a Spherical-Waves Model for Mutual Coupling. International Journal of Electronics and Communications, accepted for publication.
[7] Mathews CP, Zoltowski MD. Eigenstructure techniques for 2-D angle estimation with uniform circular arrays. IEEE Trans. on Signal Processing Sept. 1994; 42-9:2395-2407. 


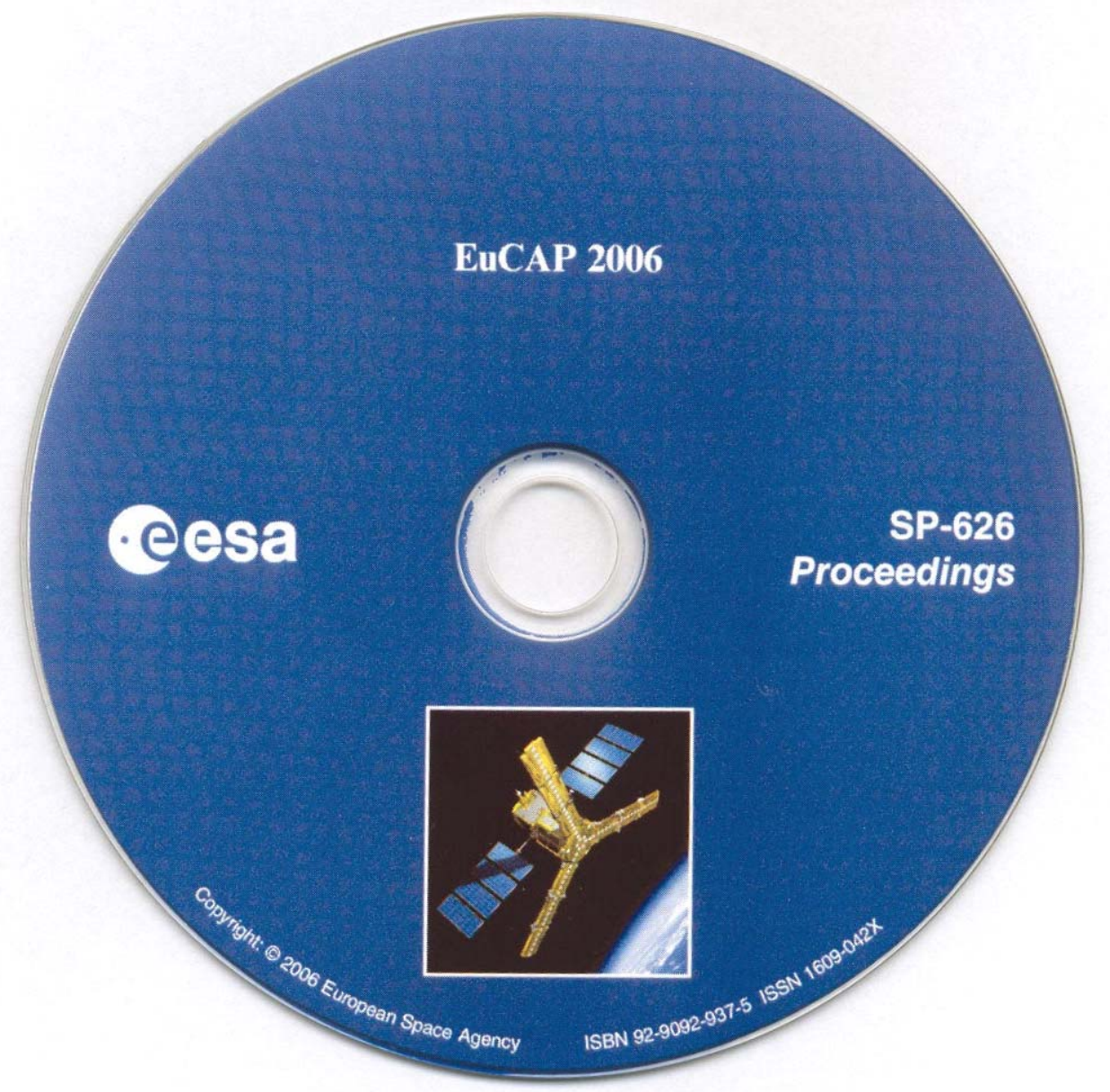




\section{EUCAP CHAIRS}

J. Mosig (General Chair), EPFL, CH

P. Ingvarson (Vice Chair), Saab Ericsson Space, $S$

B. Arbesse r-Rastburg (Vice Chair), ESA-ESTEC, NL

A. Roederer (Honorary Chair), ESA-ESTEC, NL

P. Brachat (Local Chair), France Telecom R\&D, F

C. Pichot (Local Chair), Univ. de Nice, F

Publication

Editors

Published by:

Price:

ISBN No:

ISSN No:

Copyright:
Proceedings of the European Conference on Antennas and Propagation 'EuCap 2006', Nice, France (ESA SP-626, October 2006)

H. Lacoste \& L. Ouwehand

ESA Publications Division

ESTEC

Postbus 299

2200 AG Noordwijk

The Netherlands

The Netherlands

60 Euros

92-9092-9375

1609-042X

(c) 2006 European Space Agency 
SP-626

October 2006

\author{
Proceedings of
}

\title{
The European Conference on Antennas and Propagation: EuCAP 2006
}

\author{
6-10 November 2006 \\ Nice, France
}

European Space Agency Agence spatiale européenne 